\title{
EP-195
}

\section{Cystic metastasis from an adenocarcinoma of pancreas: A case report}

\author{
Nattawut KEERATIBHARAT*
}

Department of Surgery, Suranaree University of Technology, Nakhon Ratchasim, Thailand

Introduction: A 75-year-old female was presented to our hospital due to obstructive jaundice. Computed tomography demonstrated a pancreatic head mass that causing common bile duct and intrahepatic duct dilatation without evidence of advanced disease. She was underwent pyloric-preserving pancreatoduodenectomy and they discharge without complication. Although the incidence of hepatic cystic liver metastasis from pancreatic cancer is extremely rare, care should be taken to when a cystic liver lesion was detected with pancreatic cancer.

Methods: In operative finding, we found the 1-cm liver cyst at segment 5 and excisional biopsy was done. The pathological report revealed well-differentiated adenocarcinoma of head of pancreas without nodal metastasis and cystic metastasis well-differentiated adenocarcinoma of liver.

Results: Then we reviewed the imaging study, it showed a small cystic lesion without enhancing portion at liver segment 5 that mimics intrahepatic duct dilatation and a cystic lesion at segment 6 that previously seen in preoperative imaging study. The patient underwent chemotherapy with Gemcitabine. Six months after surgery, the computed tomography revealed increase sized of cystic lesion at segment 6 and development of ascites. Ten months after surgery, the patient was dead due to severe septicemia from urinary tract infection. The previous study show a range of spectrum of hepatic cystic liver metastasis with histopathologic confirmation of diagnosis. Conclusions: Although the incidence of hepatic cystic liver metastasis from pancreatic cancer is extremely rare, care should be taken to when a cystic liver lesion was detected with pancreatic cancer. 